UCRL-ID-144099

\title{
Final Report for the PolyHANAA
}

J.B. Richards, P.L. Stratton, W.J. Benett, R.P. Koopman, F.P. Milanovich

\section{April 30, 2001}

U.S. Department of Energy

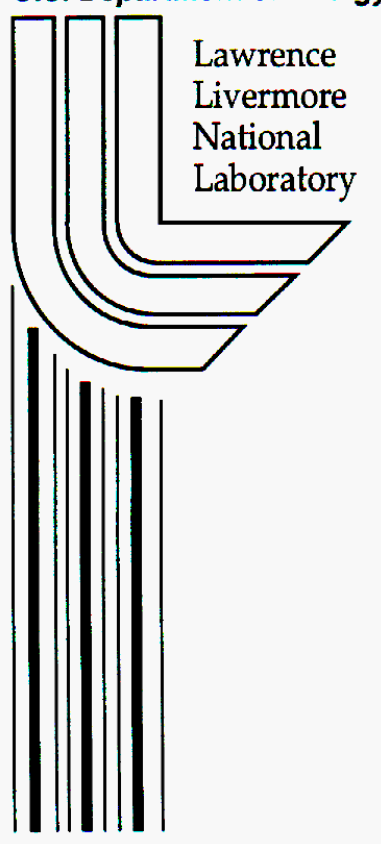




\section{DISCLAIMER}

This document was prepared as an account of work sponsored by an agency of the United States Government. Neither the United States Government nor the University of California nor any of their employees, makes any warranty, express or implied, or assumes any legal liability or responsibility for the accuracy, completeness, or usefulness of any information, apparatus, product, or process disclosed, or represents that its use would not infringe privately owned rights. Reference herein to any specific commercial product, process, or service by trade name, trademark, manufacturer, or otherwise, does not necessarily constitute or imply its endorsement, recommendation, or favoring by the United States Government or the University of California. The views and opinions of authors expressed herein do not necessarily state or reflect those of the United States Government or the University of California, and shall not be used for advertising or product endorsement purposes.

This work was performed under the auspices of the U. S. Department of Energy by the University of California, Lawrence Livermore National Laboratory under Contract No. W-7405-Eng-48.

This report has been reproduced directly from the best available copy.

Available electronically at http://www.doe.gov/bridge

Available for a processing fee to U.S. Department of Energy and its contractors in paper from

U.S. Department of Energy

Office of Scientific and Technical Information

P.O. Box 62

Oak Ridge, TN 37831-0062

Telephone: (865) 576-8401

Facsimile: (865) 576-5728

E-mail: reports@adonis.osti.gov

Available for the sale to the public from

U.S. Department of Commerce

National Technical Information Service 5285 Port Royal Road

Springfield, VA 22161

Telephone: (800) 553-6847

Facsimile: (703) 605-6900

E-mail: orders@ntis.fedworld.gov

Online ordering: http://www.ntis.gov/ordering.htm

OR

Lawrence Livermore National Laboratory

Technical Information Department's Digital Library

http://www.llnl.gov/tid/Library.html 


\section{Final Report for the PolvHANAA}

\section{Deliverable for Nucleic Acid System - PCR, Multiplex Assays and Sample Preparation Project Task 1 and \\ 24-Chamber PCR Device Improvements, Characterization, and}

\section{Testing Project}

J. B. Richards, P. L. Stratton, W.J. Benett, R. P. Koopman,

F. P. Milanovich

April 30, 2001 


\section{Table of Contents}

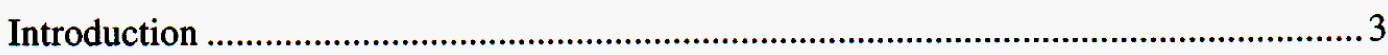

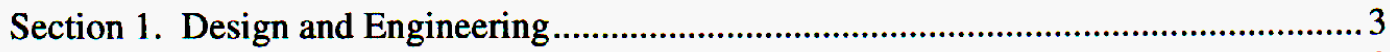

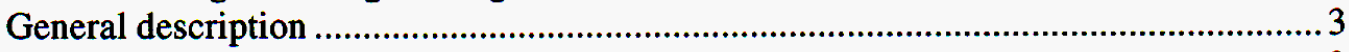

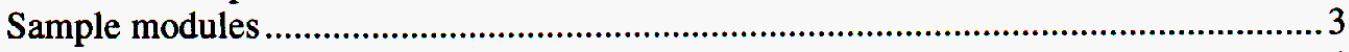

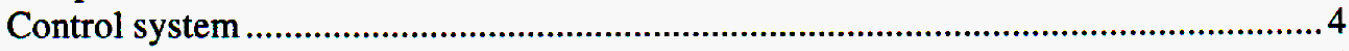

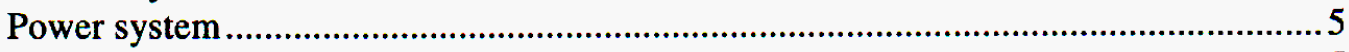

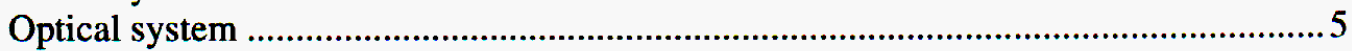

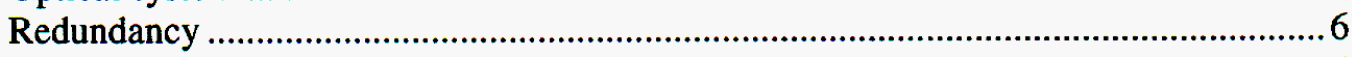

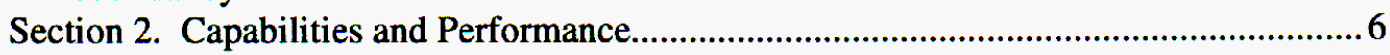

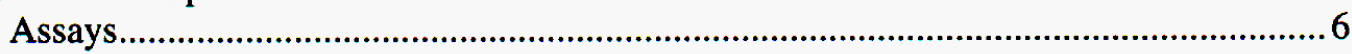

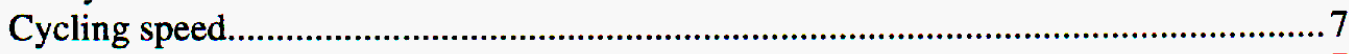

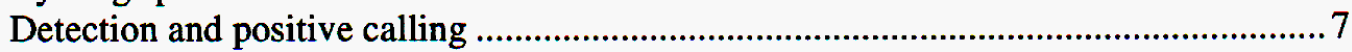

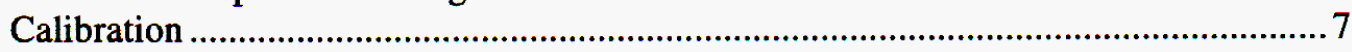

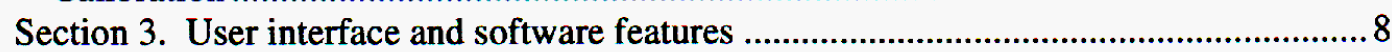

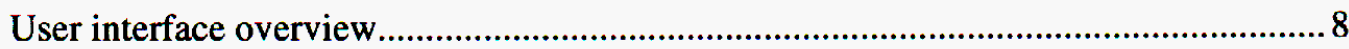

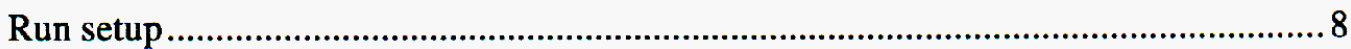

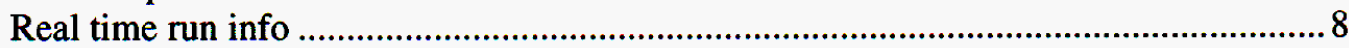

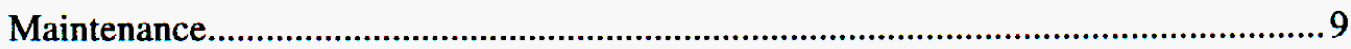

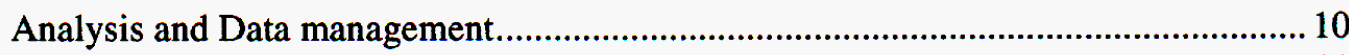

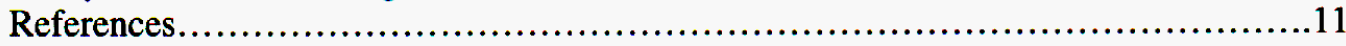

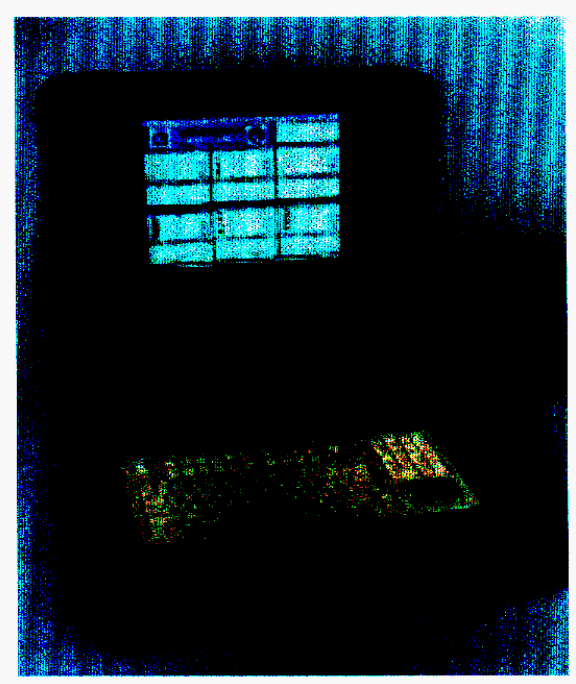

The PolyHANAA 


\section{Introduction}

The PolyHanaa is a 24 chamber thermal cycling instrument designed to perform rapid, realtime optical detection of biological agents using the Polymerase Chain Reaction (PCR) process with TaqMan ${ }^{\mathrm{TM}}$ reagents. Liquid samples are pipetted into small, disposable polypropylene tubes that are then inserted into the chambers. The 24 duplex chambers are divided into 6 independent groups and run information is presented simultaneously, in real time, for all groups.

\section{Section 1. Design and Engineering}

\section{General description}

The PolyHANAA is packaged in a carry on size suitcase measuring $24 \times 18 \times 10$ inches. The cover contains a 15" flat panel color display with a built in tilt mechanism. The control buttons for the panel are discretely placed to the left of the panel in a recessed array. There is a compartment below the panel that contains a place to store thermocouples. The tray of the suitcase contains a full size keyboard with touch pad, power connection, on/off switch, fuse housing and Ethernet connection. The 24 chambers are arranged in a row at the back of the tray, with labels for chamber number and a label for group number which is attached to the captured covers which prevent stray light from entering the chambers during runs. In front of each group is a recessed thermocouple connector that is used during temperature calibration. The front side of the suitcase contains ports that can be used for external connections to a keyboard, mouse or monitor. There is also a recessed button to select one of two internal processors to be active.

\section{Sample modules}

Each of the 24 chambers are contained in a separate, $1 \mathrm{~cm}$ wide sample module (see Figure 1) which is extractable using a small, red pry bar on the top. These modules are removable and interchangeable between chamber positions. Each module is identical to the modules used in the HANAA ${ }^{1}$. In addition to the spring loaded mechanism which positions the silicon micro chambers, each module contains all of the optical detection circuit except the silicon detector. Electrical connections are made through 5 pins that extend out the front of the modules. The two top pins are for the platinum heater and the bottom three pins provide power to the two LEDs at the back of the sample module.

Figure 1. A PolyHANAA sample module, shown with filter assemblies removed.

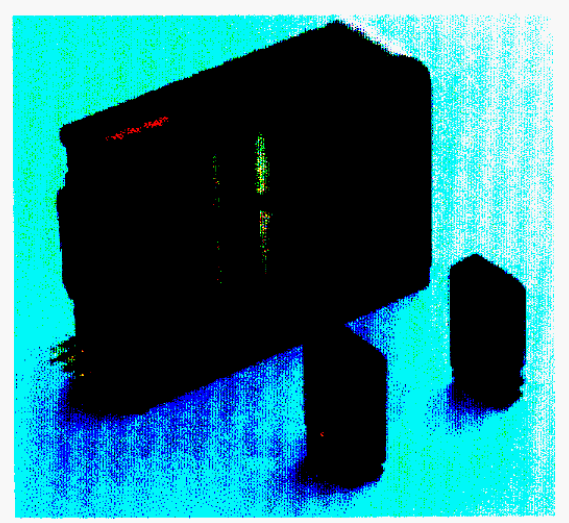


The silicon chambers are also identical to those on the HANAA ${ }^{1}$. They are advanced thermal cyclers, with a series of cooling vents to allow for efficient cooling from the fans, which are connected to the module by a ducting system positioned between and below the modules. The extremely low mass cyclers are heated by platinum resistance heaters, which are deposited onto the silicon surface. The platinum also serves as a thermometer in that its resistance is a function of its temperature. This resistance is continuously monitored and used in a feedback loop to control thermal cycling.

The sample vials are made of PCR compatible polypropylene and contain 25 microliters of liquid. In general, 20 microliters of this is master mix and 5 microliters is actual sample. The sample vials are hexagonal in cross section to match the silicon etch planes that define the internal volume of the thermal cyclers. The inserts contain captured covers that snap closed to prevent loss of liquid during PCR. The walls of the insert that face the windows in the silicon chamber are flat.

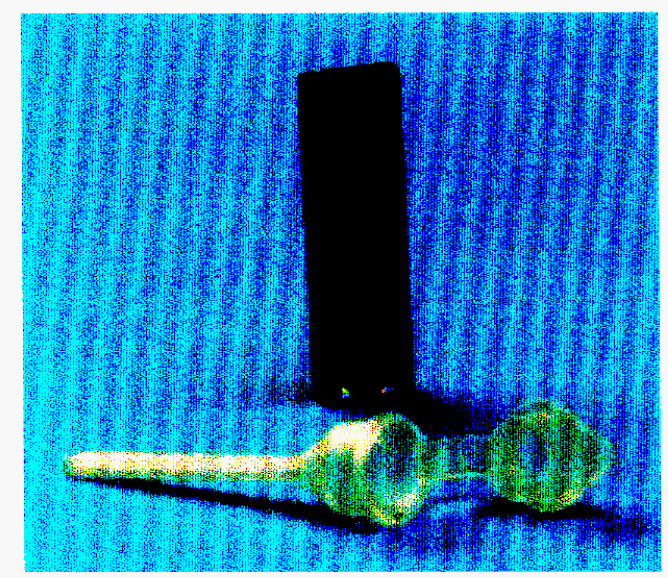

Figure 2. Photo of advanced cycler with insert

\section{Control system}

The control system for the sample modules is identical to that of the HANAA ${ }^{1}$. Each group on the PolyHANAA is treated like a separate HANAA. Chambers can be run in the fast mode using TAG (touch and go) points or in a slower mode by setting the TAG multipliers to unity such that the TAG points match the hold points and therefore have no effect. As explained in the manual ${ }^{2}$, the TAG points precede each hold setpoint and allow the control system to heat/cool the silicon chamber to a higher/lower temperature to allow for the slower thermal response of the water compared to the silicon. The result is extremely fast thermal profiles with exceptionally sharp transition regions. 
Each group has several boards associated with control and detection. The primary board is a Quad Heater Controller that contains the microcontroller circuitry. In addition, there is an Octal Detector Board, an Octal Photodiode Integrator Board and a Power Conversion board. These boards provide for loading settings to any of the modules as well as collecting data from them during runs or during maintenance operations. The control loop operates at 20 cycles per second. The boards communicate with the primary user interface software through four PCOS ('PC on a stick') microprocessors via two serial ports.

\section{Power system}

The PolyHANAA is powered from a 110 VAC wall outlet. The AC power is converted to 12 VDC to operate the instrument. While no battery system is built in, it would be possible to run the PolyHANAA from an external battery supply. In addition to providing power for thermal cycling, computers, monitor, etc., power is also used to drive chassis fans, which keep the internal electronics cool and stable during operation.

\section{Optical system}
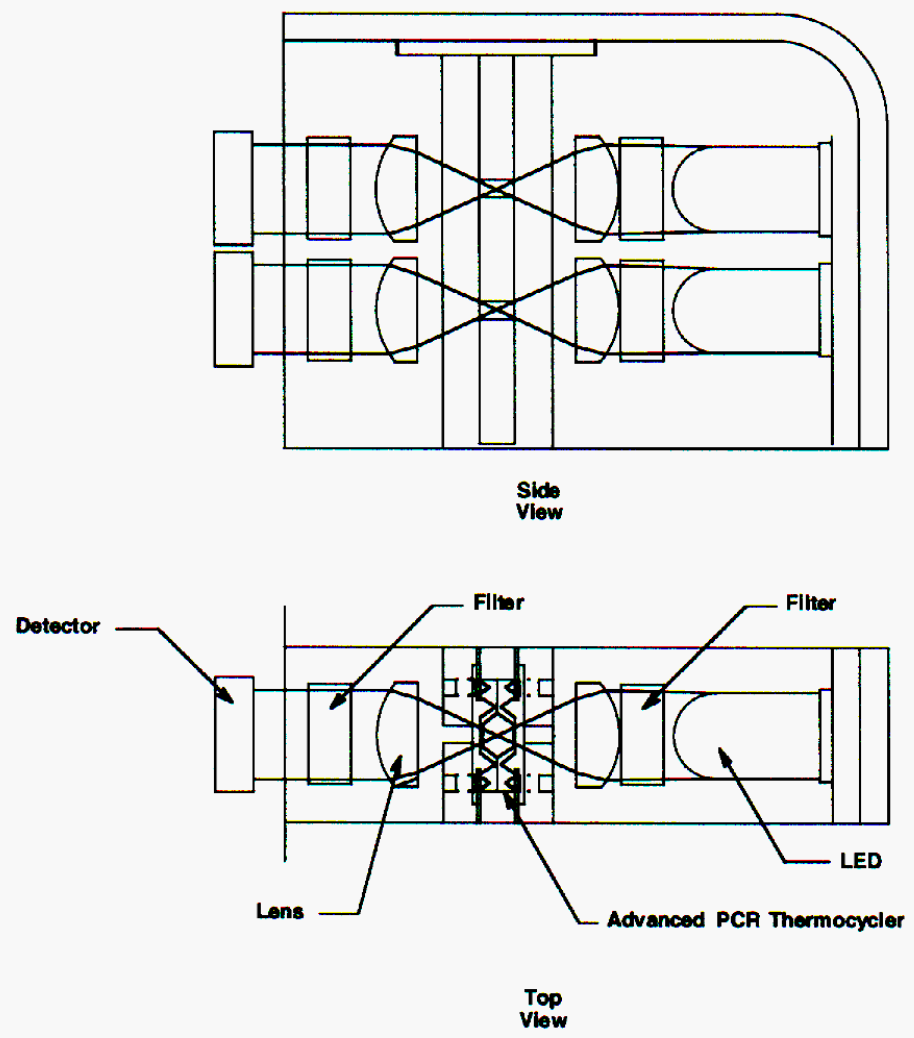

Figure 3. PolyHANAA optical system

The optical system for the PolyHANAA, shown in Figure 3, is identical to that of the HANAA $^{3}$. It incorporates a high power blue and a high power green LED within each module. Each LED has a one-quarter inch square clean-up bandpass filter in front of it to prevent any longer wavelength LED radiation from overwhelming the relatively weak 
fluorescence signal. The excitation filters are designed to pass from 460 to $500 \mathrm{~nm}$ (blue) and from 515 to $545 \mathrm{~nm}$ (green). Standard plano-convex lenses focus the light into the chamber and collect it on the other side.

The emission filters for the blue LED (channel B) are optimized for FAM and are designed to pass between 520 and $550 \mathrm{~nm}$. The channel A filters are designed to pass between 565 and $605 \mathrm{~nm}$. This is optimized for JOE emission, but would also work well for TAMRA. (FAM, JOE, and TAMRA are fluorescent tags of different wavelengths attached to DNA probes.) The filters are removable and can be changed, providing the user has purchased the desired filter from an outside vendor.

All four filter locations actually have another filter in addition to the interference filter already described. This is a Schott absorbing glass filter to eliminate long wavelengths (BG-39 for blue, BG-40 for green) and very short wavelengths (OG-515 Ch. B, OG-570 Ch. A) from reaching the detector.

The filter holders need to be positioned such that the square output windows face the chamber. If this is true, the two colored dots (one for each filter holder) will be on the same side of the module, facing the user when the connection pins are facing to the left.

\section{Redundancy}

The original intention for the PolyHANAA was to have biaxial redundant power and control circuitry. However, as the prototype was delivered, the two independent, internal Pentium processors are not interchangeable. While the active processor (the one shown on the display) can be selected by the external recessed button, the B processor can not be used to operate the PolyHANAA control system due to failure of an electronics board. This board could not be repaired within the time and budget constraints of the project. At present, the B processor can only serve as a separate processor for saving files or running other applications. Full implementation of biaxial redundancy could be accomplished in the next version of this instrument.

Ports are available for plugging in an external monitor, keyboard or mouse if any of the primary units fail. As for power system redundancy, if one of the two active power supplies fails, there will be enough power to run at least 12 chambers on the remaining power supply with no action required on the part of the user.

\section{Section 2. Capabilities and Performance}

\section{Assays}

Each chamber supports a duplex assay. The dual optical detection systems are optimized for detecting FAM in the B channel and JOE or TAMRA in the A channel. Interchangeable optical filters make it possible to customize the system for other dyes. Performance is increased with the use of dark quenchers. This reduces the amount of background signal and increases the instrument's dynamic range. 


\section{Cycling speed}

With TAG points, rise times are approximately 4 seconds and fall times are approximately 17 seconds. This leads to a minimum cycle time of 21 seconds when cycling between 57 and 94 degrees Centigrade. Figure 4 shows curves for 3 different hold times and how precise the transition regions are.

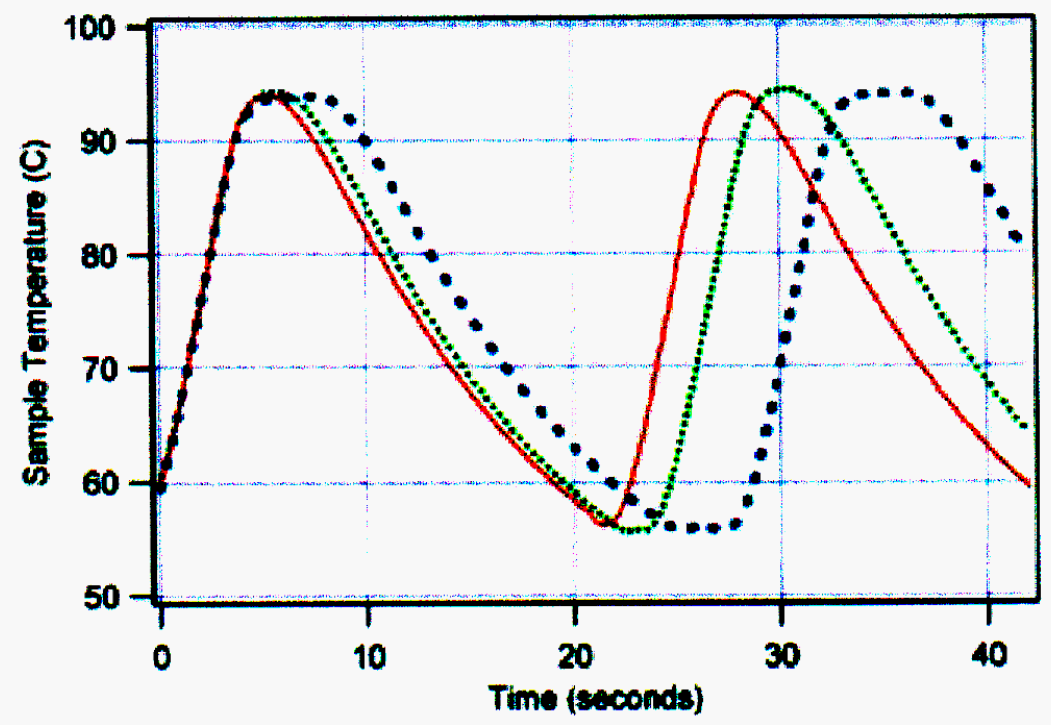

Figure 4. Liquid profile overlay demonstrating 'thermal agility'

\section{Detection and positive calling}

Raw signals from the photodiodes are collected throughout the run after each cycle. The signal level is converted to a number between 0 and 1023 by an analog to digital converter. The value for each cycle is normalized to the level obtained on the tenth cycle. In addition, a constant is added to the value for each cycle. The constant's value is equal to the difference between 200 and the raw signal level for cycle 10 . This is done to eliminate the effect of varying amounts of leakage in the optical system from one chamber to another.

CT (threshold cycle) values between calibrated chambers can vary up to $+/-2$ cycles. For any one chamber, the CT value should remain within $+/-1$ cycle.

\section{Calibration}

Chambers can be calibrated by connecting the type $\mathrm{K}$ thermocouple to the recessed connector in front of each group. The sensing end of the thermocouple has a holder that positions the sensor inside a standard sample vial at the proper depth. The vial should contain 25 microliters of water or water with oil to avoid evaporation. Between one and six groups can be calibrated at one time. Each calibration run takes approximately 6 minutes. 


\section{Section 3. User interface and software features}

\section{User interface overview}

Together with the large number of chambers, it is the user interface on the PolyHANAA that separates it from the HANAA and allows substantial operational versatility. A large amount of information is presented in real time for all 48 assays simultaneously. Data analysis and management capability is extensive and straightforward.

The user interface for the PolyHANAA is written using the application Igor Pro (WaveMetrics, Inc., Lake Oswego, OR). This application is ideal for the PolyHANAA in that it provides sophisticated capabilities in control operations, user interactions and analysis as well as publication quality graphical capabilities.

Users interact with the PolyHANAA via buttons on permanent panels or by pull-down menus. The main menu bar contains a combination of Igor Pro's built-in menus and LLNL designed menus. The LLNL menus have a 'p.' prefix and appear on the right side of the menu bar. In addition to the menu bar, users can execute specific commands on the Igor Command Line, which is at the bottom of the Command Window, which appears in the upper right hand corner of the display. The command line is a normal input mode for Igor but isn't essential to operate the 24 chamber unit. A history of previously executed commands is retained in the upper portion of the Command Window above the Command Line.

This section of the report covers features associated with various operational subsystems of the user interface. A detailed explanation of all the user menus is in the manual ${ }^{2}$.

\section{Run setup}

Users enter setup parameters to define each PCR run. Each group has a thermal profile, consisting of the high and low hold temperatures and the corresponding hold times. The number of cycles is also entered. The current values for a given group can be quickly verified using the 'profile' button on the group's panel. Templates can also be defined to quickly enter a profile from a look-up table. Integration times are also defined for each group.

Each chamber is assigned an assay name or the name 'not used' if the assay is to be simplex. The assay names come from either a popup list of user defined labels or can be typed in directly. A run is defined as a group of groups cycling through PCR together, including from 1 to all 6 of the groups.

\section{Real time run info}

At the start of a run, an automatic run number is assigned with the format MMDDYY\#. This run number appears in the group's title bar. At the start of the run, the right side of the panel will turn green and all assay boxes will be yellow. If an assay is determined to be positive, the yellow box will turn red and the CT value will appear in the History window. If the sample was named as a positive control, the box will turn pink (positive, but 
expected). At the end of the run, any non-positive chambers are called negative and turn green. Unused chambers are gray throughout the run.

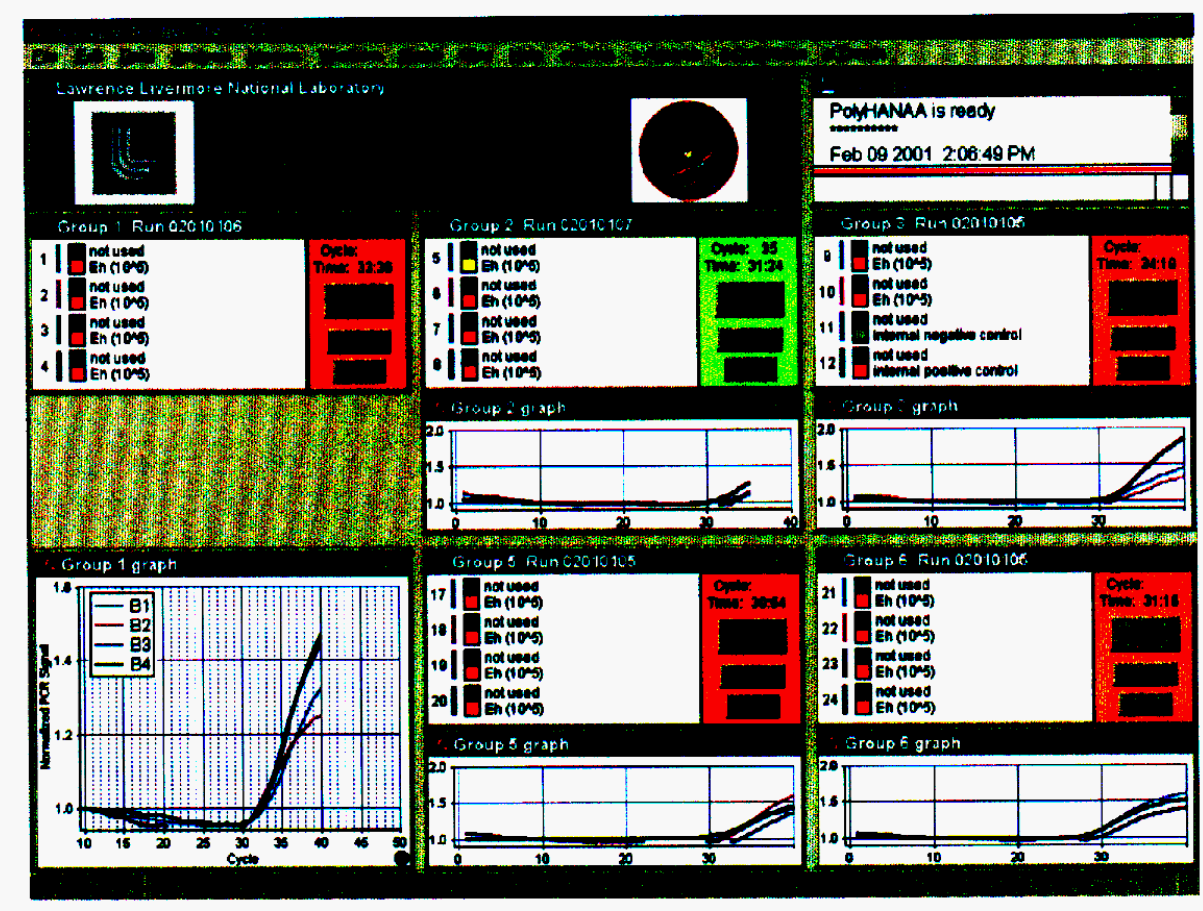

Figure 5. Real-time information displayed during run mode

The graph associated with the group shows the normalized diode signals after each cycle. Chamber traces are color coded per the vertical bars next to each chamber number on the group's panel. Channel A traces are dashed and channel B traces are solid. The 'mag' button on the panel gives an enlarged graph, plus legend, that will appear above or below the group until the (now renamed) 'norm' button is selected. Note that the graph displaces another group's information and will be overwritten if that other group is also running.

A run can be stopped at any time by individually stopping all of the groups involved. Using the 'Stop All' button will stop all runs. A group that is stopped will have the right side of the panel change color from green to red. The same thing will happen when a run goes to completion. In that case, a red ' $F$ ' will appear after the indicator showing the number of cycles in the run.

The time value below the cycle number corresponds to the number of the last cycle that was completed (as opposed to the cycle number the cycler is currently on).

\section{Maintenance}

Calibration is the primary maintenance operation. The user has the option of seeing a realtime graph of the calibration routine. Only two graphs can be monitored at a time; one from 
side A (groups 1-3) and one from side B (groups 4-6). After a group is calibrated, an automatic layout is available to document the result (see Figure 6).

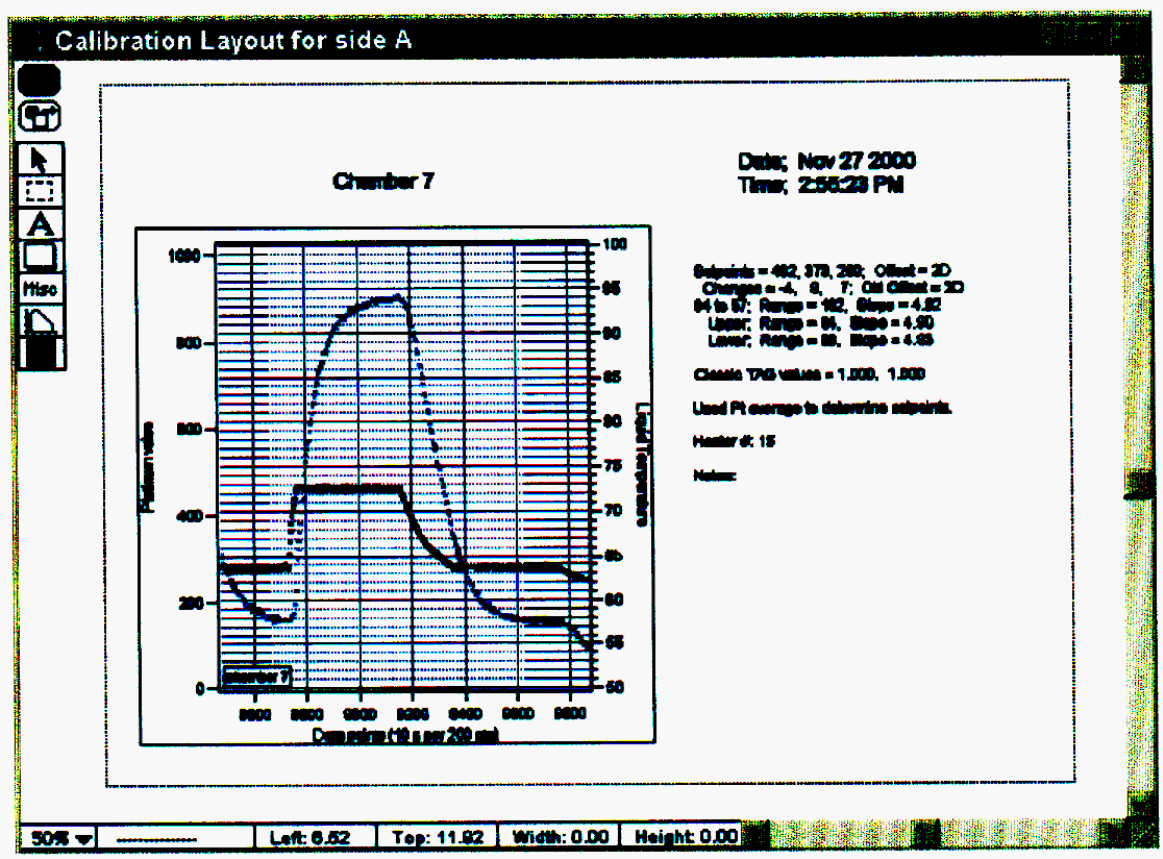

Figure 6. Automatically generated layout of calibration results

Trends are another form of real-time plot that display liquid temperature vs. time. Several types of trends are supported. Some allow real-time modification of TAG points or calibration levels. Another operates as a strip chart recorder, where the temperature will be plotted for whatever chamber the thermocouple is sampling, whether the chamber is in the same group as the thermocouple or not.

When the PolyHANAA is first turned on, the software checks each chamber to determine if it is operational and alerts the user in the History window. Other maintenance functions include a diode test function to read diode values and a similar function to read current values of the thermocouples.

\section{Analysis and Data management}

The 'p.Analysis' menu provides analysis functions that can be used whenever the PolyHANAA is not performing PCR thermal cycling in the run mode. The primary functions of the analysis menu are to review old data, to test the positive calling algorithm, to check signal levels or CT values and to create overlay plots.

The analysis menu takes advantage of Igor's built in system of data folders, where each data folder contains data from a given run, including setup information as well as detection 
data. Run data can be reprocessed using different positive calling parameters to tune to a particular assay or to see why positives were called when they were. The original run data is not changed in this process so the data folder will always contain values that were on the original run.

In addition to analysis functions supplied by Lawrence Livermore, Igor Pro has an exhaustive suite of analysis functions, curve fitting, derivatives, etc. that can easily be used under the Analysis menu on the left side of the menu bar. Igor Pro also has the best graphing package available, with the ability to add any annotations and make a graph look any way desirable. Virtually every aspect of the graph is user adjustable through interactive dialog panels.

Whenever a run finishes or is stopped, the data are automatically saved. A data folder is automatically created within Igor's root directory and an archive file is created outside Igor, on the desktop. A separate Igor file is also available for saving old data folders such that the local directory doesn't get filled up. This other experiment file only contains the software supporting data analysis, so it is much smaller and more efficient.

The user manual for the PolyHANAA is incorporated into the Igor Pro help system. It is accessible via the Help Browser under the Windows menu. The manual explains all the special menu items. It also has a collection of Operations Guides, helpful tips and warnings. Lastly, it contains a troubleshooting guide and recovery procedures to help with problems that a user may encounter.

\section{References}

1). "User Manual for LLNL's 24-Chamber Instrument (a.k.a. PolyHANAA)", Jim Richards, UCRL-ID-143441

2). "Miniaturized detection system for handheld PCR assays", Richards et. al., Proceedings of SPIE, Biochemical and Bimolecular Sensing, 4200, pp. 64-73

3). "Handheld Advanced Nucleic Acid Analyzer", Benett et. al., Proceedings of SPIE, Biochemical and Bimolecular Sensing, 4200, pp. 55-63 